\title{
Ratschläge in der Alltagskommunikation: Zur Verwendung einer sedimentierten Form im Englischen
}

\section{Einleitung}

In diesem Beitrag beschäftigen wir uns mit linguistischen Formen zur Realisierung von Ratschlägen in englischen Alltagsgesprächen. Der Fokus liegt auf einem der häufigsten Formate zum Erteilen eines Ratschlags im Englischen, nämlich „Why don’t you X?“. Besonders spannend an diesem Format ist die Tatsache, dass es nicht nach dem Grund für ein Nicht-Handeln fragt, sondern stattdessen eine bestimmte Handlung zur Lösung eines Problems empfiehlt. Unsere Hypothese ist, dass es sich um eine sedimentierte Form des alltäglichen Erteilens eines Ratschlags handelt. Im Folgenden präsentieren wir Evidenzen hierfür und fragen nach dem Vorteil einer solchen Formulierung, zumal andere, direktere Formen für das Ratschlagen wie z. B. „Do X!“ oder „You should do X“ auch zur Verfügung stehen. Aber zuerst wenden wir uns der Frage zu, was Alltagsratschläge sind.

\section{Was zählt als ein Ratschlag im Alltagsgespräch?}

Nach unserem Verständnis liegt ein Ratschlag im Alltagsgespräch dann vor, wenn eine Sprecherin eine Lösung für etwas anbietet, das als ein Problem der Gesprächspartnerin behandelt wird. ${ }^{1}$ Die Gesprächspartnerin kann ihr Problem selbst als ein solches formulieren und um Rat bitten, oder die Sprecherin kann ein latentes Problem bei der Partnerin diagnostizieren und dafür eine Lösung anbieten.

Im folgenden Gesprächsausschnitt liegt die erstere dieser zwei Möglichkeiten vor: Stan ruft seine Schwester Joyce an, um explizit um einen Rat zu bitten:

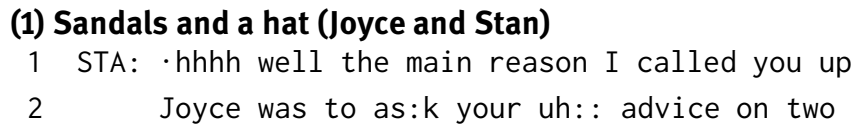

\footnotetext{
1 In diesem Beitrag benutzen wir die weibliche Form generisch sowohl für diejenige, die einen Rat ausspricht, als auch für die Rezipientin dieses Ratschlags. Allerdings sind Ratgebende und Ratrezipierende in unseren Alltagsdaten nicht immer Frauen. Ähnliches zeigen auch die Arbeiten von Heritage \& Sefi (1992) und Stivers et al. (2017).
} 


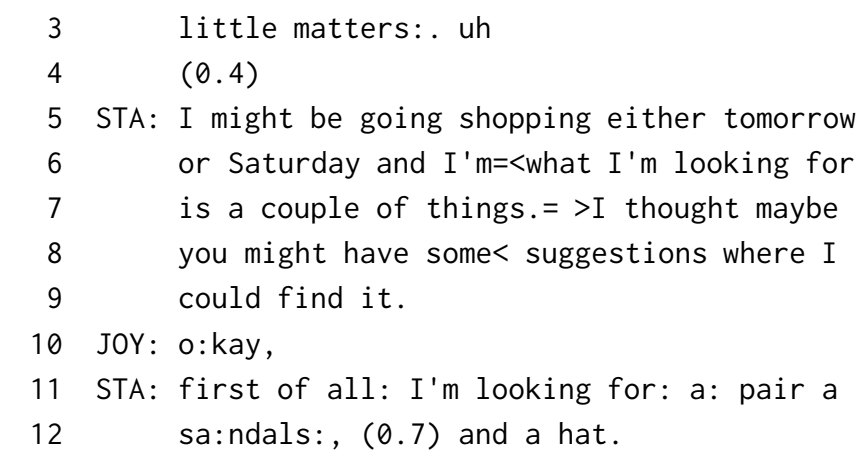

((einige Turns später - Stan beschreibt, welche Art Sandale er sucht))

13 STA: something that's comfortable:, that a<

14 that will la:st, and that you know (0.8)

15 looks: fairly decent, but<=

$16 \rightarrow$ JOY: $=\uparrow$ why don't you go get s:om:e (.)

17 STA: and for the ha: $t$, I'm looking for

18 somethi:ng uh a little different,

((einige Turns später - Joyce berichtet über den Kauf eines Huts für einen gemeinsamen Freund))

19 STA: hhhh well $\uparrow$ where can I find something

20 like that Joyce. I mean a $\uparrow$ good hat.

21 youknow I don't care paying ten dolla:rs

22 or so ${ }^{\circ}$ or even more.

23 JOY: [(pt)

24 STA: [you know a $\uparrow$ good ha: $t$, [something that

25 JOY: $\quad[(($ sigh $))$

26 STA: would look< something that I'd-

$27 \mathrm{u}:$ [I'd have a variety of things

28 $\rightarrow$ JOY: [个why don' $t$

29 STA: to loo:k at [:,

30 $\rightarrow$ JOY: [个why don't you: go into

$31 \rightarrow \quad$ Westwoo: $d,(0.4)$ and go to $\uparrow$ Bullocks.

$32 \quad(1.2)$

33 STA: Bullocks., you mean that one right $u: m$

34 (1.1) tch! (.) right by the: u:m (.)

35 what's it the plaza theatre: :?=

36 JOY: =uh huh,

$37 \quad(0.4)$ 
38 STA: ${ }^{\circ}($ memf : : )

39 JOY: ${ }^{\circ}$ yeah,

40 STA: why that Bullocks. is there something

41 about it?

42 JOY: they have some pretty nice things. and

43 you could probably find one you like

44 there,

((einige Turns später, nachdem weiter über Bullocks geredet wird))

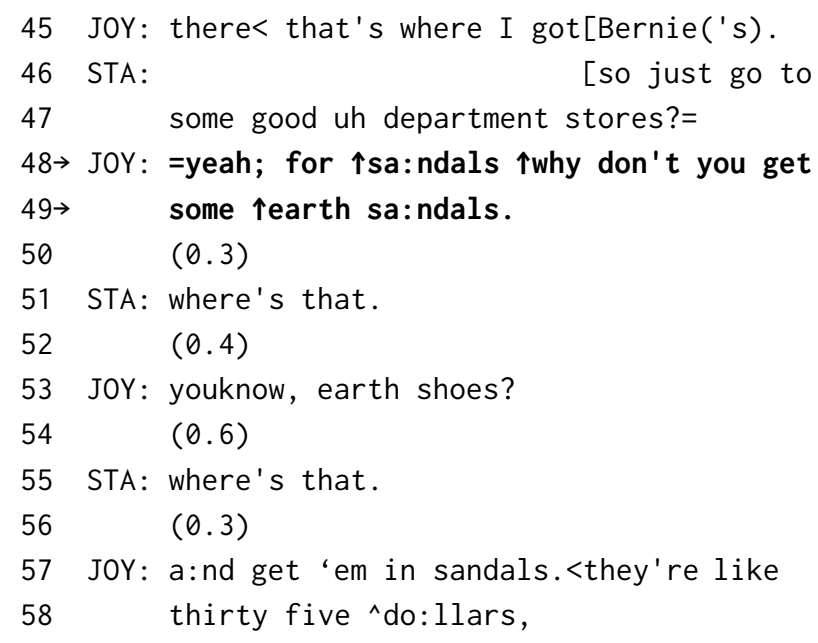

In den Zeilen 1-3 gibt Stan als Grund seines Anrufs den Wunsch an, von Joyce eine Empfehlung darüber zu bekommen, wo er am besten Sandalen und einen Hut kaufen kann (Z. 11-12). Schon während er beschreibt, welche Sandalen er genau sucht, beginnt Joyce einen Turn, der als Ratschlag formuliert ist: = $\uparrow$ why don't you go get s:om:e (Z.16). Stan fährt aber mit seiner Beschreibung seines Wunschhuts fort (Z. 17-18 und nach einer Einschubsequenz Z. 20 ff.).

In den Zeilen 19-20 erneuert Stan seine Bitte um einen Vorschlag, woraufhin Joyce folgenden Rat für den Hut erteilt: 个why don't you: go into Westwoo:d, (0.4) and go to 个Bullocks (Z. 30-31). Auf Stans Nachfrage hin (Z. 40-41) erklärt Joyce, warum sie gerade Bullocks empfiehlt (Z. 42-44). Einige Turns später liefert sie dann einen Vorschlag für den Kauf von Sandalen: for $\uparrow$ sandals $\uparrow$ why don't you get some 个earth sa:ndals (Z. 48-49).

In dieser Ratschlagsequenz also wird der Rat vom Gesprächsteilnehmer explizit erbeten und von der Sprecherin erteilt, indem sie dreimal die Formulierung „Why don't you X“ verwendet. Ein Rat kann aber auch ohne eine explizite Bitte danach erteilt werden. Dies findet vor allem dann statt, wenn eine Gesprächsteil- 
nehmerin ein persönliches Problem darstellt (troubles talk) oder sich über etwas beschwert (complaint) (vgl. Günthner 1996, 2006). Im folgenden Ausschnitt aus einem Telefongespräch zwischen zwei Schwestern, Lottie und Emma, kommt eine solche Situation vor:

(2) Stay down-1 (nb009-4)

((Lottie wohnt in Newport Beach südlich von Los Angeles, während ihre Schwester Emma in einer Großstadt nördlich davon wohnt, wo sie unter der schlechten Luftqualität leidet. Emma hat aber eine Ferienwohnung in Newport Beach, wohin sie sich wochenends zur Erholung begibt.))

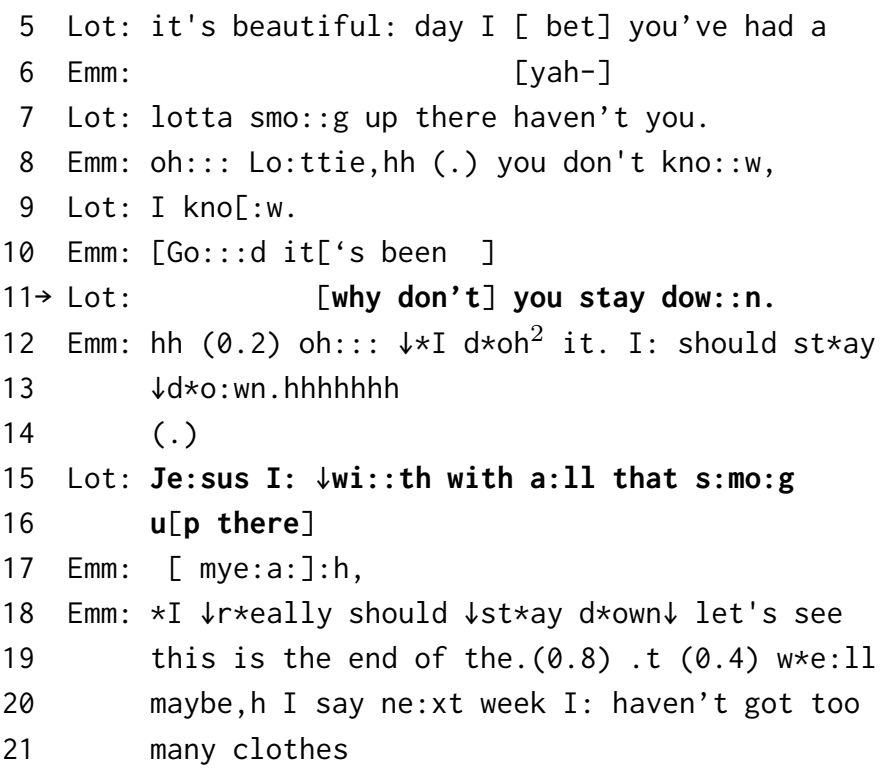

In den Zeilen 8 und 10 beginnt Emma, sich über den Smog in der Großstadt zu beschweren: oh::: Lo:ttie .hh (.) you don't kno::w...Go:::d it's been. Bevor sie fortfahren kann, bietet Lottie eine Lösung für ihr Problem an: Emma soll einfach in Newport Beach bleiben: why don't you stay dow::n (Z. 11). Dass Lotties Turn als Ratschlag verstanden wird, sehen wir an Emmas Antwort darauf. Sie gibt zu, dass das eine Lösung wäre: oh::: I know it. I: should stay dow:n (Z. 12-13). Lottie fügt nun eine Begründung für ihren Vorschlag hinzu: Je:sus I: wi::th with a:ll that s:mog up there (Z. 15-16), woraufhin Emma nochmals beteuert, dass sie unten bleiben sollte (Z. 18). Allerdings lässt die Art und Weise, wie Emma fortfährt, vermuten,

2 Das Wort wird nasal ausgesprochen. Gemeint ist vermutlich know. 
dass sie doch nicht länger bleiben wird, zumindest nicht an diesem Wochenende (Z. 20-21).

Anhand dieser zwei Beispiele können einige Hauptmerkmale einer Ratschlagshandlung im Alltagsgespräch ausgemacht werden: ${ }^{3}$

i. Die Handlung erfolgt als eine Erwiderung auf eine initiierende Handlung, sei es eine Bitte um Rat, sei es eine Problemdarstellung (troubles talk) bzw. Beschwerde (complaint) seitens der Gesprächspartnerin.

ii. Eine solche initiierende Handlung etabliert einen Kontext, in dem ein Ratschlag relevant gemacht wird: In Anlehnung an Butler et al. (2010) bezeichnen wir einen solchen Kontext als advice-implicative.

iii. Die Ratgebende schlägt eine Handlung oder Handlungsabfolge vor, die das Problem der Rezipientin lösen bzw. lindern soll. Impliziert wird, dass dies der Rezipientin Vorteile bringen wird, wenn sie die empfohlene Handlung ausführt.

iv. Die Ratgebende wird positioniert als eine Person, die Erfahrung bzw. Expertise in der Angelegenheit hat und die weiß, was für die Rezipientin am besten ist.

v. Von der Rezipientin wird erwartet, dass sie sich zum Ratschlag Stellung nimmt und gegebenenfalls in Aussicht stellt, die empfohlene Handlung bzw. Handlungsabfolge auszuführen.

\section{Ziel dieser Arbeit und Vorgehensweise}

In diesem Beitrag wollen wir uns nun speziell mit dem in den Beispielen (1) und (2) belegten Format eines Ratschlags beschäftigen, nämlich Why don't you... (WDY). Woher können Gesprächsteilnehmer wissen, dass ein solcher Turn einen Ratschlag anbietet und nicht genuin nach dem Grund eines Nicht-Handelns beim Gegenüber fragt? ${ }^{4}$ Wir wollen argumentieren, dass WDY eine formelhafte und sedimentierte Form darstellt, deren Hauptfunktion darin besteht, Handlungen bzw. Handlungsabfolgen zu empfehlen, die dem Gegenüber hinsichtlich eines Problems Abhilfe schaffen sollen. ${ }^{5}$

$3 \mathrm{Zu}$ Ratschlägen in institutionellen Kontexten siehe auch Kallmeyer (2000) und Wegner (2016). 4 Beide Varianten werden mit fallender Intonation am Ende realisiert. $\mathrm{Zu}$ weiteren W-FrageFormaten, die keine „echten“ Fragen darstellen, siehe Egbert/Vöge (2008) und Günthner (1996, 2006, 2010).

5 Unsere Daten liefern ebenso Evidenz für andere Verwendungen von WDY, z. B. um Einladungen auszusprechen oder um Instruktionen zu erteilen. In diesem Beitrag beschränken wir uns aber 
Unsere Datenbasis besteht aus transkribierten Audio- und Videoaufnahmen von natürlich zustande gekommenen Gesprächen im britischen und amerikanischen Englisch, sowohl am Telefon als auch im face-to-face-Dialog. Die Analyse beruht auf einer Kollektion von 48 WDY-Turns in diesen Gesprächen, die in advice-implicative Kontexten vorkommen und von den Rezipienten als Ratschläge behandelt werden. Für die in diesem Beitrag gewählten Beispiele haben wir die von Gail Jefferson entwickelten Transkriptionskonventionen mit normalisierter Orthografie beibehalten (s. Jefferson 2004 und Hepburn/Bolden 2013).

Im Folgenden präsentieren wir zuerst die Evidenz, die uns zur Sedimentierungshypothese geführt hat. Anschließend fragen wir nach dem Vorteil des WDYFormats für das Ratschlaggeben in englischsprachigen Alltagsgesprächen.

\section{Why don't you... als sedimentierte Form des Ratgebens}

Es gibt verschiedene Hinweise in unseren Daten, dass WDY eine formelhafte und sedimentierte Form des Ratgebens ist.

\subsection{Formale Restriktionen (constraints)}

Ein erster Hinweis auf die Sedimentierung dieser Form ist die Tatsache, dass sie nicht mit jedem beliebigen Verb verbunden werden kann. Das Verb ist zwar lexikalisch frei, es muss aber eine dynamische Handlung (mit Agens) ausdrücken. Ein nicht-dynamisches Verb wie z. B. like, wenn verbunden mit why don't you, wird auch in einer Beschwerdesequenz als eine genuine Frage nach dem Grund verstanden (s. hierzu auch Bolden/Robinson 2011). Dieser Verwendung findet sich z. B. im folgenden Gesprächsausschnitt, bei dem drei Frauen über eine Bekannte reden.

(3) „Why don't you like her“ (Bolden, p.c.)

1 RUT: I don't like her at all

2 MAD: do you talk to her ever?

auf advice-implicative Kontexte, in denen WDY eine Handlung bzw. Handlungsabfolge als Lösung eines Problems beim Gegenüber vorschlägt.

6 Wir bedanken uns bei Galina Bolden (Rutgers University), die uns auf diesen Fall aufmerksam gemacht hat. 
4 RUT: rarely.

$5 \rightarrow$ MAD: so why don't you $\operatorname{lik}(h)$ e her.

6

(0.3)

7 RUT: ${ }^{\circ}$ she's too nice ${ }^{\circ}$

Das Verb like verweist hier auf eine nicht-dynamische, statische Handlung; to like someone ist etwas, das nicht willentlich gemacht werden kann. Deshalb, obwohl Ruth sich über die Bekannte beschwert, kann Maddies Erwiderung in Z. 5 nicht als die Lösung von Ruths Problem verstanden werden, etwa ,Ich empfehle dir, sie zu mögen'. Stattdessen wird Zeile 5 als eine Bitte um Erklärung für einen z. Zt. geltenden Zustand behandelt, nämlich Ruths Nichtmögen dieser Frau. Im nächsten Turn liefert Ruth hierfür eine Erklärung, indem sie behauptet, die Bekannte sei zu nett (Z. 7). Ein WDY-Format für das Erteilen von Ratschlägen unterliegt also der Beschränkung, dass es mit einem dynamischen Verb kombiniert werden muss.

Es gibt bei diesem Format aber nicht nur Restriktionen hinsichtlich des Verbs, sondern auch hinsichtlich des Tempus und des Aspekts des Verbs. Das Verb muss in der einfachen Form des Präsens sein, um einen Ratschlag zu implementieren. WDY-Formen, bei denen das Verb z. B. im Vergangenheitstempus steht, werden nicht als Ratschläge behandelt. Das sieht man im folgenden Gesprächsausschnitt: Leslie spricht mit ihrem Mann Skip am Telefon und rätselt mit ihm darüber, wer der Dieb sein könnte, der in der vorigen Nacht in ihr Haus eingebrochen ist. Skip berichtet, dass er beim benachbarten Bauern ein brennendes Licht in der Nacht gesehen habe.

(4) „Big boy“(Holt:X Christmas 2:1:6)

1 SKI: yeah. all their lights were on $I$ and (.)

2 I: (.) just took it that he was probably:

3 eh (0.8) gone dow: $n$ to: eh (.) you know

$4 \quad k$ - start milking.

$5 \quad(1.9)$

6 LES: oh: .

7 (2.8)

8 LES: oh well you $\uparrow$ should've $\uparrow$ told me that,

9 I'd've $\uparrow$ told the police: 'cause they said

10 oh laddie's got very big $\downarrow$ feet.

11 they didn't say who:,

$12(0.4)$

13 SKI: hm:, 


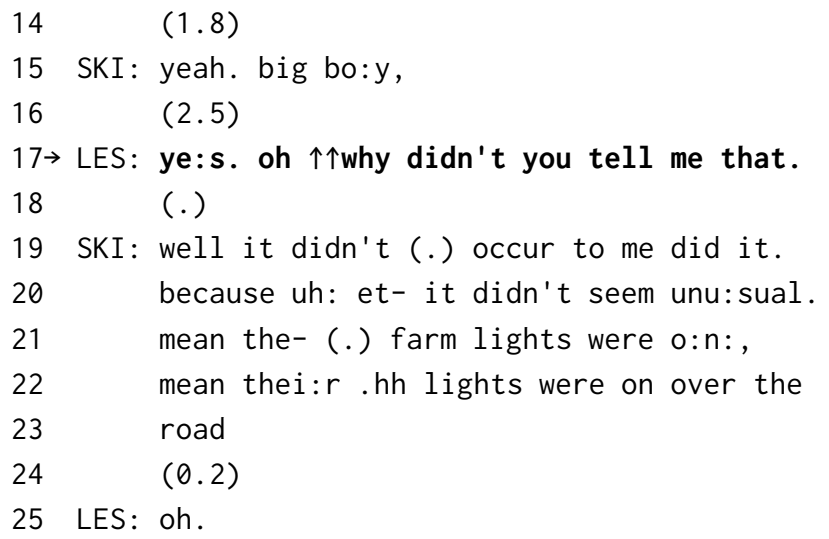

In diesem Ausschnitt geht es zwar um eine Handlung, die empfehlenswert wäre (Z. 8), aber Leslies Turn in Zeile 17 oh $\uparrow \uparrow$ why didn't you tell me that kann nicht als ein Vorschlag verstanden werden, dass Skip diese Handlung vollziehen soll. Stattdessen fragt Leslie mit ihrem Turn nach dem Grund von Skips Unterlassen dieser Handlung in der (unmittelbaren) Vergangenheit, was er im nächsten Turn damit begründet, dass er es nicht für wichtig genug erachtet hat (Z. 19-23). ${ }^{7}$ Eine negative why-Frage, die das vergangene Verhalten eines Gegenübers thematisiert, kann also nicht als ein Ratschlag in unserem Sinne verstanden werden. Wenn etwas schon geschehen bzw. nicht geschehen ist, ist es sinnlos, es als Lösung eines Problems zu empfehlen. Das Erteilen von Ratschlägen in dem hier vertretenen Verständnis ist immer zukunftsorientiert.

Das Englische bietet bekanntlich verschiedene Verbformen an, mit denen Sprecher sich auf zukünftige Handlungen beziehen können: Neben der Verwendung des Präsens in der einfachen Form (The train arrives at ten) unter anderem auch be V-ing (The train is arriving at ten) oder be going to $V$ (The train is going to arrive at ten). Aber keine dieser anderen Zukunftsformen kommt bei Ratschlägen in unseren Daten vor. Das Verb im WDY-Format ist ausschließlich im Präsens mit der einfachen Form (ohne -ing Erweiterung) in der Funktion des Ratschlags zu finden.

Auch im Hinblick auf das Subjekt gibt es beim WDY-Format Restriktionen: Für einen Ratschlag muss das Subjekt you eine Referenz auf das Gegenüber zum Ausdruck bringen. ${ }^{8}$ Eine Form wie z. B. why doesn't he... wird nicht als Ratschlag,

7 Bei Z. 17 bestehen aufgrund des prosodischen Formats funktionale Bezüge zu Vorwurfsfragen: s. Günthner 1996.

8 Es gibt allerdings eine subjektlose Variante von WDY, nämlich why not $x$ ?, die wir aber hier nicht weiter behandeln (siehe aber Thompson/Couper-Kuhlen im Druck). 
sondern als Frage nach dem Grund eines (Nicht)Handelns seitens eines Dritten verstanden:

(5) „Pop the question“ (Call Home_En 6067)

1 BAB: so wow. I hope Rabbi Grunner gets married

2 to this chick. .heh

3 ANN: I know(h) th(h)a(h)t wou(h)ld b(h)e $s(h) o$

$4 \quad h h{ }^{\circ}$ awesome ${ }^{\circ} .=<$ we'd better be invited to

5 the [wedding, that's all I could say.

6 BAB: [do you think< I know.

7 BAB: do you think (you're gonna) fly home for

$8 \quad$ it?

$9 \quad$ mhh hih hih

10 ANN: it wouldn't be< I don't think it would be

$11 \quad *$ that soon.

$12 \quad(0.4)$

13 BAB: $\downarrow$ you never know $\downarrow$, maybe he's ner- he is,

14 I mean .hhhhh

15 ANN: uh heh heh huh

16 BAB: he really should really soon, you know?

$17 \quad(0.2)$

18 ANN: I know.

$19 \rightarrow$ BAB: why doesn't he just pop the question

20 already.

21 ANN: .t [I don't] ^know: :

22 BAB: [mh heh ]

23 ANN: I hope he ^does,

24 BAB: yeah, ime $\downarrow$ too.

Auch wenn in den Zeilen 19-20 andere WDY-Eigenschaften vorliegen - z. B. ein dynamisches Verb, eine einfache Form des Präsens, eine Negation -, ist dieses why-Format nicht als ein Ratschlag an die Gesprächspartnerin Ann zu verstehen, weil es in der dritten Person formuliert ist. Stattdessen wird es als eine Frage nach dem Grund des (Nicht-)Handelns der betreffenden Person behandelt. Im nächsten Turn behauptet Ann, dass sie den Grund nicht kennt (Z. 21).

Schließlich gibt es Restriktionen hinsichtlich der Polarität eines WDY-Formats: Die Negation muss vorliegen, sonst wird ein Turn mit why - auch wenn er ein you beinhaltet - als eine genuine Frage nach dem Grund und nicht als Ratschlag verstanden. Im folgenden Fall handelt es sich um einen advice-implicative Kontext; trotzdem wird die Äußerung in Zeile 26 nicht als Ratschlag behandelt: 
(6) „Make a decision“ (Call Home_En 6067)

1 BAB: well you should. 个but Ann I think this has to

2 *stop. unless he makes a (quick) decision.

3 ANN: *you're right it does*. but like $\mathrm{I}<\mathrm{I}$ mean

4 BAB: it's not healthy.

5 ANN: I k[now,

6 BAB: $\quad[*$ for you*.

7 ANN: ibut I love being with him so mu[ch.

8 BAB: $\quad[$ (and so)

9 ANN: [it's so much fun. $\uparrow$ ]

10 BAB: [so you can< ]

11 you can still be with him,

$12 \quad(1.0)$

13 BAB: just in a different wa(h)[y. nhheh

14 ANN: [but he holds me

15 very tight.

$16 \quad(0.7)$

17 BAB: .t .hhhh

$18 \quad(0.3)$

19 ((sigh)) $\uparrow$ why does he do *that*.

20. (0.3)

21 ANN: hhhheh nhih .hhh!

22 BAB: $\uparrow$ why does he do this= <he doesn't

23 it's not fair to you.

24 ANN: I know, *it's not fair to me at all.*

$25 \quad(0.5)$

$26 \rightarrow$ BAB: so why are you let- ((distorted))

27 ANN: it's very unlike him. you know,

Babs' Frage so why are you let- (Z. 26), welche höchstwahrscheinlich auf dem Weg $\mathrm{zu}$,why are you letting him (do this)‘ war, stellt keinen Vorschlag für Anns zukünftiges Handeln dar, sondern fragt nach dem Grund ihres jetzigen Handelns.

Es ist wichtig festzuhalten, dass ein WDY-Format (mit Negation) nicht dazu beiträgt, nach dem Grund eines vorher festgestellten Nicht-Handelns des Gegenübers zu fragen. Im Beispiel (1) ist es nicht so, dass Stan schon gesagt hätte, dass er nicht nach Westwood gehen will. Im Beispiel (2) hat Emma nicht bereits gesagt, dass sie nicht in Newport Beach länger bleiben will. Wäre dies der Fall und hätten die Sprecherinnen jeweils nach dem Grund dafür fragen wollen, müssten sie sagen ,Why are you not going into Westwood?' bzw. 'Why are you not staying down?'. Das heißt, es wäre eine andere aspektuelle Form des Präsens zu verwenden. In 
den tatsächlich vorkommenden Situationen führt aber die jeweilige Sprecherin das Einkaufen in Westwood bzw. das Längerbleiben in Newport Beach als eine neue Idee ins Gespräch ein: Sie regt jeweils an, dass Stan nach Westwood zum Einkaufen gehen soll bzw. dass Emma länger bleiben soll, um den Smog in der Großstadt zu vermeiden. Das WDY-Format wird von den Interagierenden/von der Rezipientin also nicht wortwörtlich interpretiert, wenn es in einem advice-implicative Kontext geäußert wird - dies liefert Evidenz für seine Sedimentierung.

\subsection{Keine wortwörtliche Bedeutung}

In der Tat ist ein WDY-Format, wenn es zum Erteilen eines Ratschlags benutzt wird, eher idiomatisch zu verstehen: Die einzelnen Bestandteile des Ausdrucks haben nicht ihre konventionelle Bedeutung. Für sonstige why-Fragen geht man davon aus, dass der propositionale Gehalt präsupponiert wird: ,Why do you always leave the fridge door open?` setzt als wahr voraus, dass der Adressat ständig die Tür zum Kühlschrank auflässt (Levinson 1983:184). Wie Günthner (1996) bemerkt, kann eine solche Frage oft als Vorwurf verstanden werden - mit der Implikation, dass die Sprecherin diese Handlung als moralisch verwerflich betrachtet. Aber auch dann wird die Handlung selbst als Tatsache behandelt. Mit einer wortwörtlich zu verstehenden why-Frage fragt die Sprecherin also nach dem Grund eines als wahr präsupponierten Handelns. Analog dazu, mit einer negativen why-Frage (,Why don't you ever close the fridge door?') fragt die Sprecherin nach dem Grund eines präsupponierten Nicht-Handelns: Eine solche Frage setzt als wahr voraus, dass die erwähnte Handlung noch nicht vollzogen wurde.

Ein Ratschlag, der mit einem WDY-Format geliefert wird, ist allerdings nicht als eine wortwörtlich negative why-Frage zu verstehen. Why don't you go into Westwood bedeutet nicht etwa ,Warum hast du entschieden, nicht nach Westwood zu gehen', sondern vielmehr ,Ich empfehle Dir, nach Westwood zu gehen'. Ähnlich bedeutet Why don't you stay down nicht etwa ,Warum hast du entschieden, nicht länger zu bleiben', sondern ,Ich empfehle dir, länger zu bleiben‘. Mit anderen Worten hat ein WDY-Format eine deontische Interpretation: Es sagt etwas über die Verpflichtung des Gegenübers aus, etwas in der Zukunft zu tun (Stevanovic/Peräkylä 2012: 316). ${ }^{9}$

Dass WDY-Formate, die Ratschläge liefern, tatsächlich so verstanden werden, sehen wir an der Art ihrer Erwiderung im Gespräch. Im Beispiel (2) antwortet Em-

9 Nach Stevanovic/Peräkylä (2012: 316) heißt „deontic“ in diesem Zusammenhang, dass eine Äußerung „[can be understood] as conveying obligations to the hearer." Siehe auch Deppermann (2006) zu ,deontischen“ Infinitiven. 
ma oh I know it. I should stay down (Z. 12-13). Das heißt, sie behandelt Lotties Turn als eine Aussage über etwas, das sie machen soll. Um mit Shaw/Hepburn (2013: 348) zu sprechen, akzeptiert Emma Lotties beratende Handlung (action) [sie akzeptiert, dass ihr eine Verpflichtung zugeschrieben wird], obwohl sie deren Inhalt (content), das Längerbleiben, höchstwahrscheinlich nicht in die Tat umsetzen wird (siehe den weiteren Verlauf ihres Turns).

Im folgenden Ausschnitt kommt es zu einer etwas anders gearteten Antwort auf einen WDY-Ratschlag:

\section{(7) „Leave off the meat“ ( $\mathrm{Nb}$ 014-9)}

((Emma leidet unter der Hautkrankheit Psoriasis. Bud ist Emmas Ehemann.))

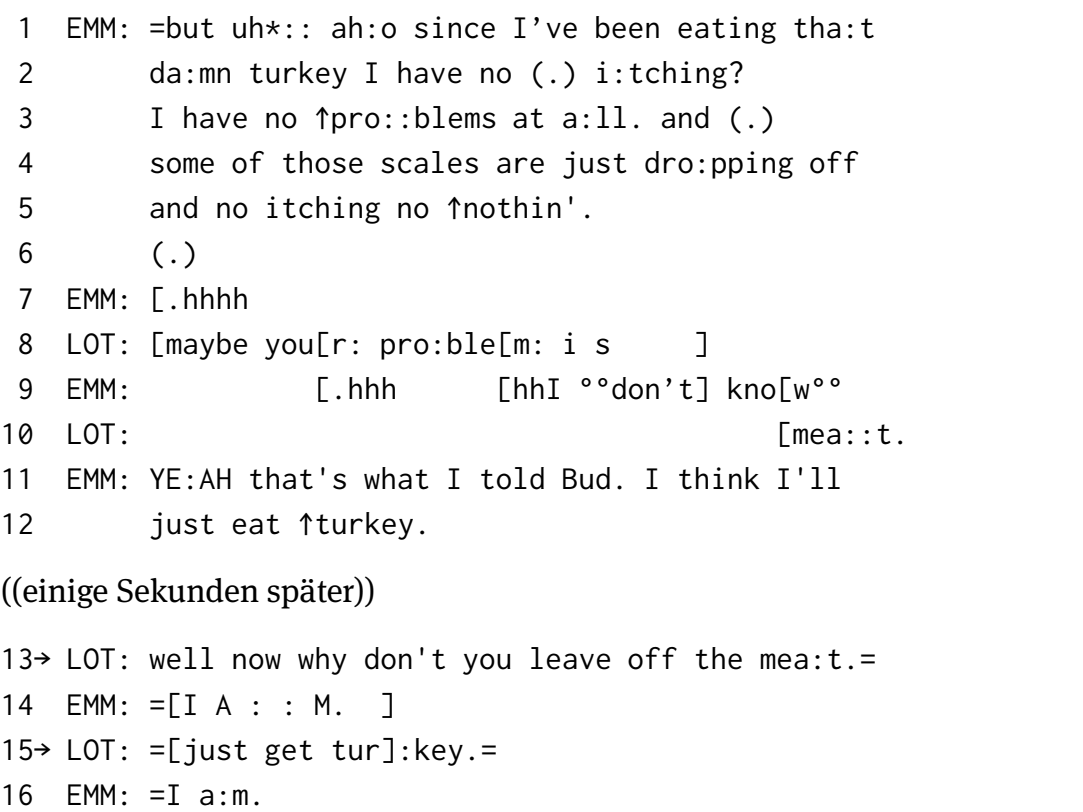

In diesem Beispiel hat Emma eigentlich schon angedeutet, dass sie vorhat, auf den Verzehr von ,Fleisch' (gemeint ist Schweine- und Rindfleisch) zu verzichten (Z. 11-12). Lotties Ratschlag in Zeile 13 well now why don't you leave off the mea:t muss also als Ermunterung verstanden werden, genau das zu tun. Emmas Antwort $I$ a:: $m$ in Zeile 14 behandelt Lotties Turn nicht als eine Frage, weshalb sie nicht auf Fleisch verzichten soll, sondern als eine positive Empfehlung, auf ,Fleisch` zu verzichten. Mit ihrer Erwiderung gibt Emma zu verstehen, dass sie schon dabei ist, den Inhalt von Lotties Ratschlag in die Tat umzusetzen (Z. 16). Das heißt, sie akzeptiert die Verpflichtung, die Lotties Turn impliziert, behauptet aber, dass sie selbst auf die Lösung gekommen ist. Die erhöhte emotive Betei- 
ligung, mit der Emma auf Lotties Vorschlag reagiert, führen wir darauf zurück, dass Emma schon gesagt hat, dass sie Entsprechendes vorhat (Z. 11-12).

Fassen wir unsere bisherige Argumentation zusammen: WDY-Formate in advice implicative Kontexten werden nicht wortwörtlich als Bitten nach Begründungen für nicht stattfindende Handlungen verstanden. Wäre dies der Fall, würden sie eine epistemische Interpretation verlangen, d. h., sie würden Wissen über die Gründe für bestimmte Ereignisse ansprechen. Stattdessen haben wir argumentiert, dass WDY-Formate eine nicht-wortwörtliche Lesart verlangen, nach der sie Lösungen für ein Problem des Gegenübers vorschlagen. Das bedeutet, dass sie als deontische Handlungen zu interpretieren sind: Sie sagen etwas über eine vermeintliche Verpflichtung des Gegenübers aus, etwas zu tun (Stevanovic/Peräkylä 2012). Oder um mit Shaw/Hepburn (2013:348) zu sprechen: „Giving advice imposes and prescribes that an action should be done rather than claiming that it will be done“ (Kursiva im Original).

\subsection{Phonetisch-phonologische Gestaltung}

Ein weiteres Indiz für die Sedimentierung von WDY-Formaten liegt in deren phonetisch-phonologischen Gestaltung: (a) die Art und Verteilung von Tonakzenten in WDY-Turns ist voraussagbar; (b) turneinleitendes WDY unterliegt der phonetischen Reduktion.

\section{(a) WDY und Tonakzente}

Wenn WDY als Format für einen Ratschlag dient, hat es eine erkennbare Melodie (siehe auch Sag/Liberman 1975). Auf dem Wort why liegt ein hoher Ton, was nahelegt, dass der Turn etwas Neues einführt (Couper-Kuhlen 2001, 2004). ${ }^{10}$ Der hohe Ansatz in Zeilen 30-31 vom Ausschnitt (1) ist in Abbildung 1 sichtbar (linker Pfeil); Abbildung 2 zeigt den hohen Ansatz bei WDY in Zeile 11 vom Ausschnitt (2) (linker Pfeil).

Zweitens liegt der Hauptakzent vom WDY-Turn auf dem Verb und wird auch durch einen hohen Ton realisiert, der oft sogar höher als der des Ansatzes ausfällt. In Abbildung 1 ist der Hauptakzent (in diesem Fall durch eine stark steigend-fallende Kontur realisiert) auf Bullocks, dem letzten Lexem des Prädikats go to Bullocks, zu sehen (rechter Pfeil); in Abbildung 2 befindet sich der hohe Ton des Hauptakzents auf down im Prädikat stay down (rechter Pfeil).

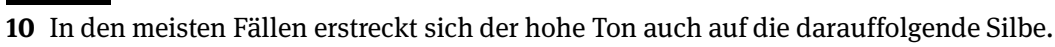




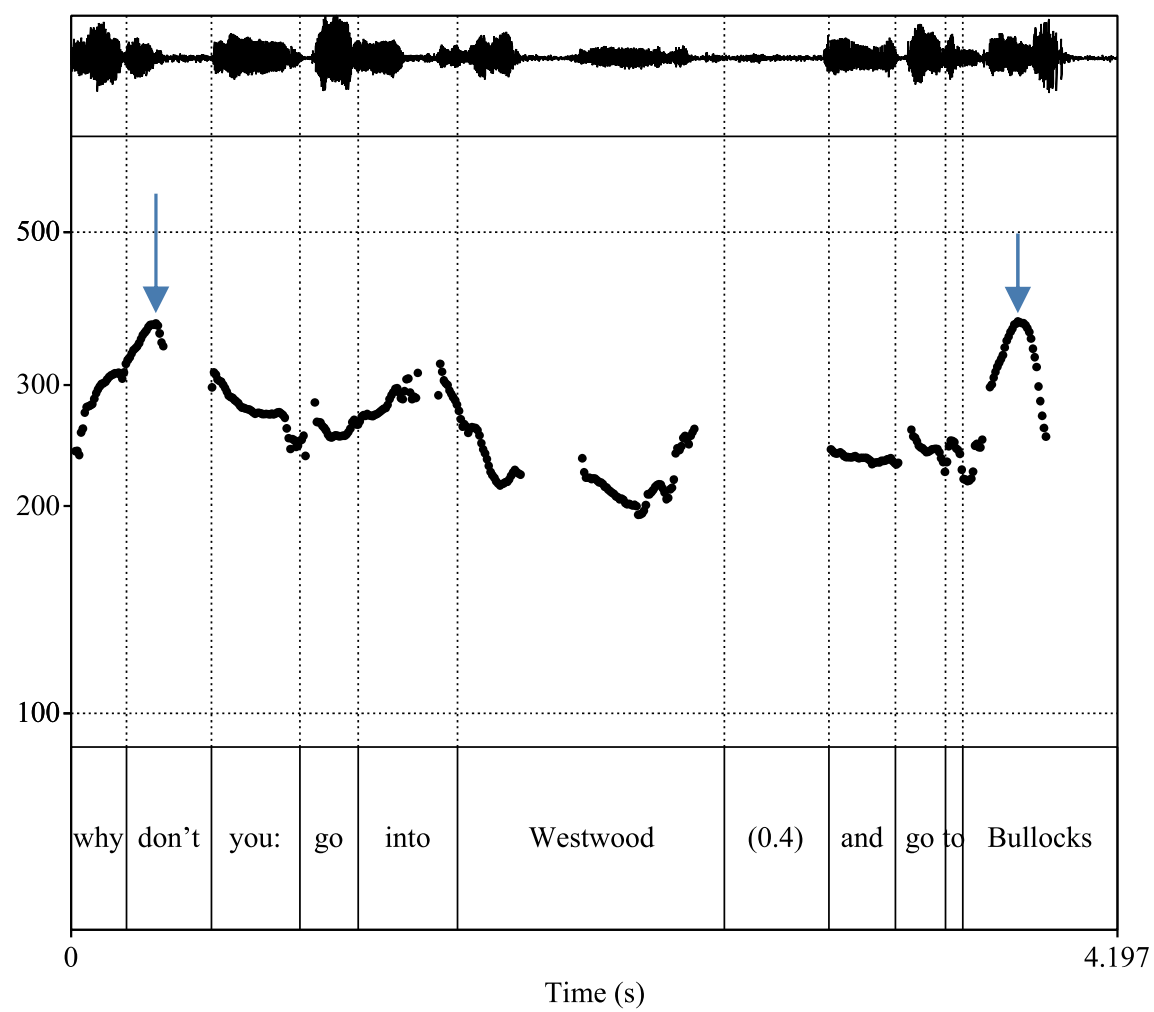

Abb. 1: Tonhöhenverlauf in Zeilen 30-31 vom Ausschnitt (1)

\section{(b) WDY und phonetische Reduktion}

Wenn WDY einen ratgebenden Turn einleitet, werden die ersten drei Wörter /war/, /doont/ und /ju/ - oft als ein Stück (chunk) produziert (Bybee 2010). In Ausschnitt (1) Zeile 30 werden die Wörter don't und you zusammengezogen zu ,dontchu'; you wird aber gedehnt, wonach es eine kleine Unterbrechung gibt, bevor das Prädikat go into Westwood folgt.

Wenn WDY als chunk artikuliert wird, gibt es nur einen Akzent auf why. Die folgenden Silben werden rhythmisch an diesen Akzent angehängt (klitisiert), wobei das /t/ von don't und das /j/ von you in der Regel zu einer Affrikate / $\mathrm{t} /$ verschmilzt und der Vokal des you zu einem Schwa /ə/ reduziert wird: /'waI doun-t $\int$ ə/. Gelegentlich können sich auch why und don't verbinden: in diesem Fall verschwindet /d/ in don't und dessen Vokal reduziert sich zu/ə/ bzw. verschwindet vollständig. Aus diesem Reduktionsprozess ergibt sich dann eine einzige Silbe: /'wam/. Das WDY Stück wird dann zu /'warn-tfə/. Das ist die Aussprache, 


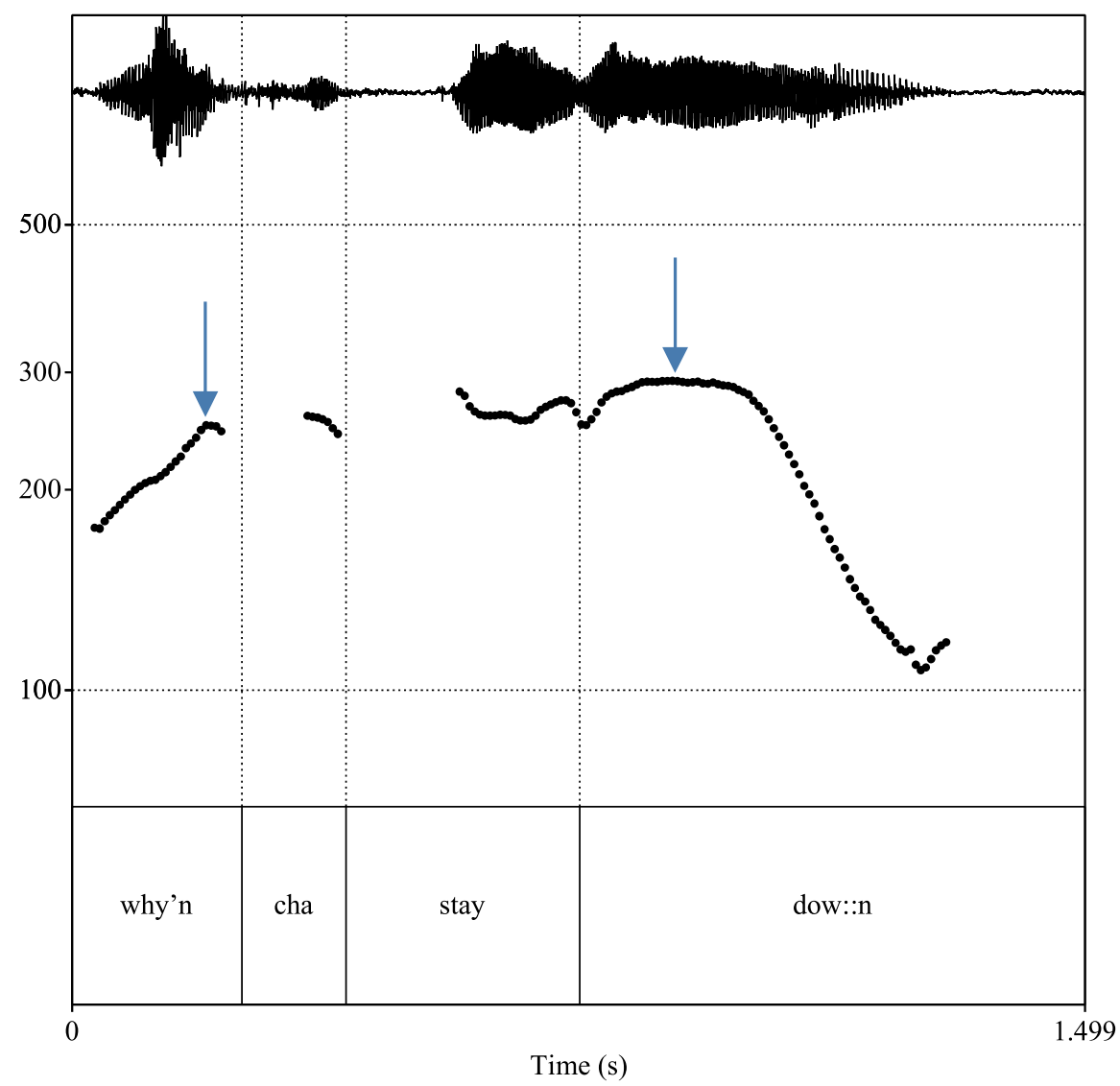

Abb. 2: Tonhöhenverlauf von Zeile $11 \mathrm{im}$ Ausschnitt (2)

die wir im Ausschnitt (2) auf why don't you stay dow::n und im Ausschnitt (7) auf why don't you leave off the mea:t vorfinden. Umgekehrt finden wir geringe oder gar keine phonetische Reduktion, wenn why don't you mit einem nicht-dynamischen Verb kombiniert wird und wortwörtlich zu verstehen ist: Das ist der Fall im Ausschnitt (3), wo so why don't you like her (Z. 5) ohne phonetische Reduktion produziert wird: /sov 'waI dount ju 'lark ər/.

Die prosodisch-phonetische Evidenz deutet also darauf hin, dass das ratgebende WDY-Format sich zu einer Konstruktion mit eigener Prosodie und Phonetik sedimentiert hat. Nach Bybee et al. (2016) ist diese Art phonetischer Reduktion ,speziell' (special), d. h., sie stellt keinen allgemeinen phonologischen Prozess dar, sondern beschränkt sich auf bestimmte lexikalische Ausdrücke - oft auf sol- 
che, die dabei sind, zu grammatikalisieren. ${ }^{11}$ Ähnliches könnte auch für die intonatorische Melodie behauptet werden, die bei ratgebenden WDY-Turns immer wieder vorkommt.

\section{Zwischenbilanz}

Wir haben versucht zu zeigen, dass englische WDY-Formate, wenn sie zum Erteilen von Ratschlägen im Alltagsgespräch benutzt werden, sedimentierte Formen darstellen. Sie unterliegen Restriktionen hinsichtlich des Prädikats, mit dem sie erscheinen; hinsichtlich des Tempus/Aspekts und des Subjekts dieses Prädikats sowie hinsichtlich der Polarität des Prädikats. Englische WDY-Formate müssen mit dynamischen Verben in der einfachen Form des Präsens mit you als Subjekt und mit der Prädikatsnegation vorkommen, um als Ratschläge verstanden zu werden. WDY-Formate sind nicht wortwörtlich zu verstehen, sondern sie haben eine idiomatische Lesart, wonach sie nicht nach dem Grund eines Nicht-Handelns fragen, sondern stattdessen eine positive Empfehlung zum Handeln liefern. Schließlich haben englische WDY-Formate eine eigene Melodie und sie enthalten phonetische Klitisierungen und Reduktionen, wenn sie Ratschläge implementieren. Aus

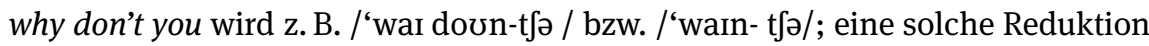
findet nicht statt, wenn why don't you für eine Frage nach dem Grund verwendet wird.

\section{WDY im Paradigma der Ratschlagsformate}

Das WDY-Format stellt nur eines unter mehreren wiederkehrenden Formaten dar, die zur Implementierung von Ratschlägen in englischen Alltagsgesprächen verwendet werden können (Couper-Kuhlen/Thompson im Druck). Unter welchen Bedingungen wählt eine Sprecherin gerade dieses Format aus? Was ist der Vorteil eines WDY-Formats? Ein Vergleich mit anderen Turnformaten für das Ratschlaggeben im Englischen kann Licht auf diese Frage werfen. ${ }^{12}$

Verglichen mit der allerhäufigsten Form des Erteilens von Ratschlägen, dem Imperativ (vgl. Deppermann i. d. B.), weist WDY zum Beispiel wichtige Unterschiede auf. Im folgenden Gesprächsausschnitt benutzt Lottie eine Imperativform, um ihrer Schwester Emma einen Rat zu geben. Der Hintergrund ist, dass Emma sich

11 Bybee et al. (2016: 427) erwähnen die why don't you-Konstruktion als einzig anderen Fall neben I don't know (Bybee/Scheibman 1999), bei dem sich der Vokal in don't zu einem Schwa reduziert. 12 Siehe auch Shaw (2013), Shaw/Hepburn (2014), Shaw et al. (2015), Couper-Kuhlen/Thompson (im Druck) sowie für den medizinischen Kontext Stivers et al. (2017). 
gerade von ihrem Mann Bud aufgrund eines heftigen Streits getrennt hat, Bud will nun nur für den Thanksgiving-Donnerstag nach Newport Beach kommen. Emma kündigt an, dass sie selbst länger dort bleiben will:

\section{(8) „Wash them out“ (Nb028-5)}

28 EMM: so I guess Bud's coming down tomorrow

$29 \mathrm{ni}: \mathrm{ght}$,

$30 \quad(0.3)$

31 LOT: [oh: . ]

32 EMM: [.hhea]hh I: GUE:SS hi-s- but he'll be here

33 Thursday but I guess he has to go ba:ck

34 Friday to go to work.h

35 LOT: ah hah,

$36 \quad(0.3)$

37 EMM: [.t

38 LOT: [will you stay di-oh well you[pro\bably<]

39 EMM: [I'M GONNA ]

40 STAY

41 .hh YOU KNOW I ONLY HA: VE one brassiere and

42 pair of panties Lottie, h

$43 \rightarrow$ LOT: well wash them ou: [:t.

44 EMM: [that's what $I(h)$ 'M DOIN

45 RI:GHT NOW I $\uparrow$ just CA:ME in,

46 LOT: oh:.

$47 \quad(0.2)$

48 EMM: .hh[hh

49 LOT: [oh: .

Nachdem Emma ankündigt, dass sie vorhat, länger in Newport Beach zu bleiben, fügt sie allerdings als Erschwernis hinzu, dass sie nicht genügend frische Unterwäsche hat (Z. 41-42). Lottie erwidert sofort well wash them out (Z. 43). Mit dieser Imperativform positioniert sie sich als Autorität, die selbstverständlich weiß, was zu tun ist, wenn man keine frische Unterwäsche hat. ${ }^{13}$ Dabei lässt sie keinen Zweifel daran aufkommen, dass dies nicht möglich sein könnte. Das heißt, sie berücksichtigt nicht, dass es eventuell Umstände geben könnte (contingencies, Curl/Drew 2008), die eine solche Handlung verhindern könnten.

13 In dieser Hinsicht gibt es hier eindeutige Bezüge zu „epistemic authority“ wie bei Heritage/ Raymond (2005) und Raymond/Heritage (2006) beschrieben. 
Im Gegensatz dazu zeigt ein WDY-Format an, dass die Sprecherin sich möglicher Hindernisse bewusst ist. Denn diese Form, obwohl in ihrer Bedeutung verfestigt, trägt trotzdem Spuren ihrer ursprünglichen wortwörtlichen Bedeutung. Wenn es Gründe gibt, die gegen das Ausführen einer mit einem WDY-Format empfohlenen Handlung sprechen, dann kann die Rezipientin diese als Begründung der Ablehnung, d.h. des Nicht-Handelns, im nächsten Turn anführen. Eine solche Entwicklung sehen wir im nächsten Ausschnitt:

(9) „Take it again“ (Geri \& Shirley)

((Shirley hat gerade erfahren, dass sie die Aufnahmeprüfung für ein Jurastudium nicht bestanden hat. Jetzt erzählt sie ihrer Freundin Geri, dass sie nicht weiß, was sie im kommenden Jahr machen soll.))

1 SHI: .t.hhhh but I really don't think I'm gonna

2 go to law school.

$3 \quad(0.3)$

4 SHI: at least not right now.

$5 \quad():$.hh

6 GER: are you se: $r$ ious, =

7 SHI: =yeh,

$8 \quad(0.2)$

9 SHI: very.

$10 \quad(0.6)$

11 ( ): .t.hh

$12 \rightarrow$ GER: .hhhh Shi:rley, I mean $\uparrow$ why don't you try

$13 \rightarrow \quad$ taking it âgai[:n.

14 SHI: [.hhh-

15 .hhhhh 'cause I really don't know if I

16 could put myself through it all over again.

$17 \quad(0.3)$

18 GER: ${ }^{\circ}$.p.t ${ }^{\circ}$ we:1l just study differently this

19 ti: [me. ${ }^{\circ}$

$21 \mathrm{SHI}$ [.t.hhh I don't kno:w it's on the Saturday

22 before final exams

$23 \quad(0.8)$

24 GER: (just) take one later than that.

$25 \quad(0.2)$

26 SHI: I ca:n't.

27 GER: why: .

28 SHI : because they don't let you. you have to take

29 it by the end of this year. 
Geris Ratschlag - angesichts der dramatischen Lage im Leben ihrer Freundin ist, dass Shirley die Prüfung wiederholen sollte: Shi::rley, I mean why don't you try taking it agai:n (Z. 12-13). Dieser Turn weist die charakteristische Melodie eines WDY-Ratschlags auf: sowohl why als auch again haben hohe Tonakzente (Abbildung 3).

Gegen Geris Vorschlag spricht aber aus Shirleys Sicht, dass sie nicht weiß, ob sie dazu den Mut hat: I really don't know if I could put myself through it all over again (Z. 15-16). Mit diesem Einwand lehnt Shirley Geris Ratschlag ab. Interessant ist, dass Shirley ihre Begründung mit einem kausalen Konnektor einführt: 'cause

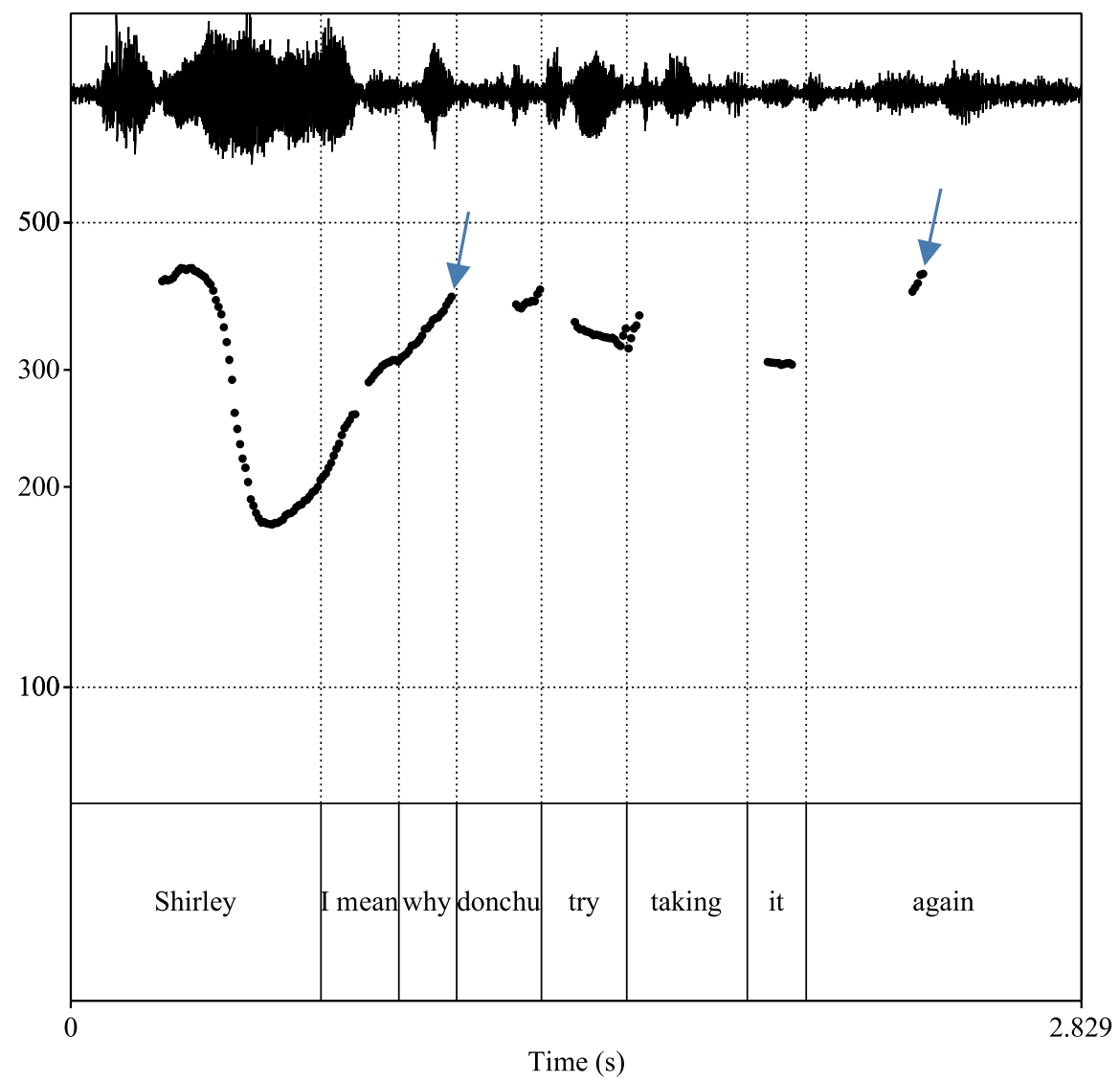

Abb. 3: Tonhöhenverlauf in Zeilen 12-13 von Beispiel (9) 
(Z. 15). Der Konnektor 'cause nimmt Bezug auf das why in Geris vorhergehenden Turn. D. h. obwohl das WDY-Format die idiomatische Lesart eines Ratschlags hat, wird formal die Möglichkeit offengelassen, dass es Gründe dagegen geben könnte. Das ist ein signifikanter Unterschied zum imperativisch formulierten Ratschlag.

Andere Ratschlagsformate im Englischen sind Deklarativa, z. B. „you couldX“. In dem folgenden Gesprächsausschnitt wird dieses Format für einen Ratschlag benutzt:

\section{(10) „Six shots“ (Nb 014-10)}

((Emmas Hautkrankheit Psoriasis hat dazu geführt, dass der Arzt ihr einen Fußnagel entfernen musste, was sehr schmerzhaft war. Jetzt berichtet sie ihrer Schwester Lottie, dass sie nicht nochmals zu diesem Arzt gehen will.))

1 EMM: ... I was going to go to my doctor up there

2 I thought I'll go: and get (.) you know let

3 hi:m then I thought oh: : god he'll wanna

4 take the other toenail off'n I don't want

5 that to come o:ff so: :,

$6 \quad(0.4)$

7 EMM: .t I was a:ll set to go do:wn here though,

$8 \rightarrow$ LOT: .t.hh we: 11 you could go dow: $n$ every SA-er

9 [THEY'RE 0-uh he's=

10 EMM: [yah.

$11 \rightarrow$ LOT: =dow: $\mathrm{n}$ there Sa:turda[y, .hh]h

12 EMM: [ye:ah.]

$13 \rightarrow$ LOT: and you only need s:ix: :=

14 EMM: =ye:ah,

$15 \quad(0.2)$

16 $\rightarrow$ LOT: uh sho::ts en: : uh this: (.) uh I didn't

17 talk to Do:ctor Nagle but I talked to his

18 hea:d nu:rse she's a

$19 \quad(0.2)$

20 EMM: .hmhh.

((die Teilnehmerinnen reden weiter über Dr. Nagles Praxis))

Lotties Ratschlag besteht darin, dass Emma jeden Samstag nach Newport Beach herunterfahren könnte/sollte, um sich dort bei Dr. Nagle Spritzen gegen ihre Krankheit geben zu lassen (Z. 8-9, 11, 13, 16). Wie Emma auf diesem Ratschlag reagiert, steht allerdings im starken Kontrast zu Ratschlägen mit WDY. Hier bestätigt sie Lotties Turns, als ob sie Aussagen wären, mit yah (Z.10) und ye:ah (Z. 12, 14). D. h. sie behandelt sie nicht als deontisch relevant. Im folgenden Verlauf des 
Gesprächs kommt es zu keiner Akzeptanz bzw. Ablehnung von Lotties Ratschlag; es ist, als ob Lottie gar keinen Ratschlag ausgesprochen hätte. Eine ähnliche Entwicklung nach einem WDY-Format wäre äußerst ungewöhnlich. Nach einem WDY-Format wird von der Rezipientin normativ erwartet, dass sie Stellung zur empfohlenen Handlung nimmt und sich ggfs. zu deren Ausführung verpflichtet oder aber Gründe angibt, warum sie das nicht tun will/ kann.

Ein WDY-Format nimmt also auf einer Deontizitätsskala eine Zwischenposition ein: Es ist etwas weniger deontisch als ein Imperativ, weil es die Möglichkeit eines Gegengrunds vorsieht; es ist aber viel deontischer als ein Ratschlag im you could-Format. Letzteres lässt eine epistemische Behandlung zu: Die Rezipientin kann den vermeintlichen Ratschlag als eine einfache Aussage (assertion) behandeln und mit ihr übereinstimmen. Beide Gesprächspartnerinnen sind sich dann darüber einig, dass die erwähnte Handlung eine Möglichkeit darstellt, wobei deren Handlungsrelevanz für die Rezipientin außer Acht gelassen wird.

Somit nimmt die als WDY verfestigte Form eines Ratschlags einen besonderen Platz im Paradigma der Ratschlagsformate ein. Sie macht es unmöglich, die deontische Komponente zu unterschlagen. Durch die Spuren ihrer ursprünglichen wortwörtlichen Bedeutung eröffnet sie gleichzeitig die Möglichkeit, Umstände einzuführen, die ein Ausführen der empfohlenen Handlung verhindern. Die Rezipientin wird dann durch äußere Umstände von der Verpflichtung befreit.

Das WDY-Format hat aber darüber hinaus eine moralische Komponente, die bei anderen Ratschlag-Formaten im Englischen fehlt. ${ }^{14}$ Diese kommt durch das Wort why zustande: Wie Robinson (2016) ausführt, hebt why die Frage der accountability hervor. Dem Gegenüber eine why-Frage zu stellen, legt nahe, dass seine Handlung bzw. sein Verhalten aus dem üblichen Rahmen fällt und somit nach einer Erklärung verlangt. Denn wenn die Handlung von sich aus verständlich wäre, bräuchte man nicht nach einer Begründung zu fragen. Bezogen auf Ratschläge mit einem why don't you-Format heißt das, dass, obwohl nicht wortwörtlich nach dem Grund eines Nicht-Handelns gefragt wird, die Sprecherin gleichwohl mit diesem Format andeutet, dass die von ihr empfohlene Handlung ,logisch` bzw. selbstverständlich wäre, sodass das Gegenüber eigentlich selbst auf die Idee hätte kommen können. Das Nichteintreten der Situation wird somit behandelt, als ob es einer besonderen Begründung bedarf. Obwohl alle gängigen Formate für das Erteilen eines Ratschlags im englischen Alltagsgespräch gewissermaßen delikate Handlungen darstellen, weil die Ratgebende sich anmaßt, zu wissen, was das Beste für das Gegenüber sei, sind Ratschläge mit einem WDY-Format besonders riskant

14 Wir sind Susanne Günthner und Jörg Bergmann zu Dank verpflichtet, die uns auf diesen Aspekt der WDY-Formate aufmerksam gemacht haben. 
für das soziale Gefüge. Denn sie lassen sich als subtile Hinweise darauf deuten, dass man das eigene Problem selbst - ohne Hilfe des Anderen - in den Griff hätte bekommen können bzw. sollen; sie versetzen die Ratsuchende damit in eine defensive Position.

\section{Fazit}

In diesem Beitrag haben wir dafür argumentiert, dass WDY ein formelhaftes und sedimentiertes Format des Englischen darstellt, mit dem unter anderem Ratschläge in advice-implicative Kontexten erteilt werden können. Die Formelhaftigkeit bzw. Sedimentiertheit des Formats (hierzu auch Auer i.d.B. und Deppermann i. d. B.) äußert sich u. a. darin, dass (a) das Format formalen Beschränkungen hinsichtlich des Verbs, des Tempus und Aspektes, des Subjekts sowie der Polarität unterliegt; (b) es eine nicht-wortwörtliche Interpretation als eine Handlung bereitstellt, die nicht nach dem Grund eines als wahr präsupponierten Nicht-Handelns fragt, sondern eine positive Handlung dem Gegenüber mit verpflichtender Tendenz vorschlägt; und (c) es systematisch mit einer erkennbaren Melodie und verschiedenen Graden der phonetischen Reduktion produziert wird.

Schließlich haben wir dafür argumentiert, dass WDY eine einzigartige Form für das Erteilen eines Ratschlags im Alltagsgespräch darstellt. Im Vergleich zu imperativischen Formen, die die Durchführung der Handlung als selbstverständlich behandeln und mögliche Kontingenzen nicht vorsehen (vgl. Deppermann i. d. B.), lässt das WDY-Format zumindest formal zu, dass das Gegenüber selbst entscheiden kann, ob es Gründe gibt, die gegen die empfohlene Handlung sprechen oder nicht. Impliziert wird, dass die Handlung als das Naheliegendste zu empfehlen ist, wenn es keine solche Gründe gibt. Das WDY-Format ist aber auf der anderen Seite präskriptiver als ein you could $X$-Format für das Erteilen des Rates, denn Letzteres lässt zu, dass die Rezipientin einfach mit der Sprecherin übereinstimmt, dass die erwähnte Handlung tatsächlich eine Möglichkeit darstellt, ohne auf ihre Handlungsrelevanz einzugehen. Das WDY-Format ist also in vielerlei Hinsicht ein differenziertes Mittel, um Freunden und Verwandten auf indirekte Weise einfache Lösungen für ihre Probleme anzubieten, wobei der Verdacht entstehen kann, dass die Betroffene selbst auf eine so einfache Lösung hätte kommen können. 


\section{Literatur}

Auer, Peter (i. d. B.): Genau! Der auto-reflexive Dialog als Motor der Entwicklung von Diskursmarkern. In: Weidner, Beate et al. (Hrsg.), Verfestigungen in der Interaktion. Konstruktionen, sequenzielle Muster, kommunikative Gattungen. Berlin: de Gruyter.

Bolden, Galina B. und Jeffrey D. Robinson (2011): Soliciting accounts with 'why'-interrogatives in conversation. In: Journal of Communication, 61(1):94-119.

Butler, Carly W. et al. (2010): Advice implicative interrogatives: Building „client-centered support“ in a children's helpline. In: Social Psychology Quarterly, 73(3):265-287.

Bybee, Joan und Joanne Scheibman (1999): The effect of usage on degrees of constituency: the reduction of don't in English. In: Linguistics, 37(4):575-596.

Bybee, Joan, Richard File-Muriel und Ricardo Napoleao de Souza (2016): Special reduction: A usage-based approach. In: Language and Cognition, 8:421-446.

Couper-Kuhlen, Elizabeth (2001): Interactional prosody: High onsets in reason-for-the-call turns. In: Language in Society, 30:29-53.

Couper-Kuhlen, Elizabeth (2004): Prosody and sequence organization in English conversation: The case of new beginnings. In: Couper-Kuhlen, Elizabeth und Cecilia E. Ford (Hrsg.), Sound Patterns in Interaction, S. 335-376. Amsterdam: Benjamins.

Couper-Kuhlen, Elizabeth und Sandra A. Thompson (im Druck): Action ascription and deonticity in everyday advice-giving sequences. In: Deppermann, Arnulf und Michael Haugh (Hrsg.), Action Ascription. Oxford: OUP.

Curl, Traci S. und Paul Drew (2008): Contingency and action: A comparison of two forms of requesting. In: Research on Language and Social Interaction, 41(2):129-153.

Deppermann, Arnulf (2006): Deontische Infinitivkonstruktionen: Syntax, Semantik, Pragmatik und interaktionale Verwendung. In: Günthner, Susanne und Wolfgang Imo (Hrsg.), Konstruktionen in der Interaktion, S. 239-262. Berlin: de Gruyter.

Deppermann, Arnulf (i. d. B.): Imperative im Deutschen: Konstruktionen, Praktiken oder social action formats? In: Weidner, Beate et al. (Hrsg.), Verfestigungen in der Interaktion. Konstruktionen, sequenzielle Muster, kommunikative Gattungen. Berlin: de Gruyter.

Egbert, Maria und Monika Vöge (2008): Wh-interrogative formats used for questioning and beyond. German warum (why) and wieso (why) and English why. In: Discourse Studies, 10(1):17-36.

Günthner, Susanne (1996): The prosodic contextualization of moral work: An analysis of reproaches in 'why'-formats. In: Couper-Kuhlen, Elizabeth und Margret Selting (Hrsg.), Prosody in Conversation, S. 271-302. Cambridge: CUP.

Günthner, Susanne (2006): Von Konstruktionen zu kommunikativen Gattungen. Die Relevanz sedimentierter Muster für die Ausführung kommunikativer Aufgaben. In: Deutsche Sprache, 34(1-2):173-190.

Günthner, Susanne (2010): Grammatical constructions and communicative genres. In: Dorgeloh, Heidrun und Anja Wanner (Hrsg.), Syntactic Variation and Genre, S. 195-217. Berlin: Mouton de Gruyter.

Hepburn, Alexa und Galina Bolden (2013): The conversation analytic approach to transcription. In: Sidnell, Jack und Tanya Stivers (Hrsg.), The Handbook of Conversation Analysis, S. 57-76. Chichester, UK: John Wiley \& Sons. 
Heritage, John und Geoffrey Raymond (2005): The terms of agreement: Indexing epistemic authority and subordination in assessment sequences. In: Social Psychology Quarterly, 68:15-38.

Heritage, John und Sue Sefi (1992): Dilemmas of advice: aspects of the delivery and reception of advice in interactions between health visitors and first-time mothers. In: Drew, Paul und John Heritage (Hrsg.), Talk at Work. Interaction in institutional settings, S. 359-417. Cambridge: CUP.

Jefferson, Gail (2004): Glossary of transcript symbols with an introduction. In: Lerner, Gene (Hrsg.), Conversation Analysis: Studies from the first generation, S. 13-31. Amsterdam: Benjamins.

Jefferson, Gail und John R. E. Lee (1981): The rejection of advice: Managing the problematic convergence of a „troubles-telling“ and a „service encounter“. In: Journal of Pragmatics, 5:399-422.

Kallmeyer, Werner (2000): Beraten und Betreuen. Zur gesprächsanalytischen Untersuchung von helfenden Interaktionen. In: Zeitschrift für qualitative Bildungs-, Beratungs- und Sozialforschung, 1:227-252.

Levinson, Stephen C. (1983): Pragmatics. Cambridge: CUP.

Pudlinski, Christopher (2002): Accepting and rejecting advice as competent peers. In: Discourse Studies, 4:481-499.

Raymond, Geoffrey und John C. Heritage (2006): The epistemics of social relations: Owning grandchildren. In: Language in Society, 35:677-705.

Robinson, Jeffrey (2016): Accountability in social interaction. In: Robinson, Jeffrey (Hrsg.), Accountability in social interaction, S. 1-44. Oxford: OUP.

Sag, Ivan und Mark Liberman (1975): The intonational disambiguation of indirect speech acts. In: Chicago Linguistic Society, 11:487-497.

Shaw, Chloe (2012): Advice giving in telephone interactions between mothers and their young adult daughters. PhD thesis, Loughborough University.

Shaw, Chloe und Alexa Hepburn (2013): Managing the moral implications of advice in informal interaction. In: Research on Language and Social Interaction, 46(4):344-362.

Shaw, Chloe, Jonathan Potter und Alexa Hepburn (2015): Advice-implicative actions: Using interrogatives and assessments to deliver advice in mundane conversation. In: Discourse Studies, 17(3):317-342.

Stevanovic, Melisa und Anssi Peräkylä (2012): Deontic authority in interaction. The right to announce, propose, and decide. In: Research on Language and Social Interaction, 45(3):297-321.

Stivers, Tanya et al. (2017): Treatment recommendations as actions. In: Health Communication, 33(11):1335-1344.

Thompson, Sandra A. und Elizabeth Couper-Kuhlen (im Druck): English why don't you $X$ as a formulaic expression. In: Ono, Tsuyoshi und Ritva Laury (Hrsg.), Fixed Expressions. Amsterdam: Benjamins.

Waring, Hansun Zhang (2005): Peer tutoring in a graduate writing center: Identity, expertise and advice resisting. In: Applied Linguistics, 26:141-168.

Waring, Hansun Zhang (2007): Complex advice acceptance as a resource for managing asymmetries. In: Text and Talk, 271:107-137.

Wegner, Lars (2016): Lehrkraft-Eltern-Interaktionen am Elternsprechtag. Berlin: de Gruyter. 